\title{
Growth Inhibition by Bupivacaine Is Associated with Inactivation of Ribosomal Protein S6 Kinase 1
}

\author{
Mushtaq Ahmad Beigh, ${ }^{1}$ Mehvish Showkat, ${ }^{1}$ Basharat Bashir, ${ }^{1}$ Asma Bashir, ${ }^{1}$ \\ Mahboob ul Hussain, ${ }^{1}$ and Khurshid Iqbal Andrabi ${ }^{1,2}$ \\ ${ }^{1}$ Department of Biotechnology, Science Block, University of Kashmir, Srinagar J $\mho K$ 190006, India \\ ${ }^{2}$ Department of Biotechnology and Bioinformatics, University of Kashmir, Srinagar Jઐ K 190006, India
}

Correspondence should be addressed to Khurshid Iqbal Andrabi; andrabik@uok.edu.in

Received 24 April 2013; Revised 14 November 2013; Accepted 18 November 2013; Published 29 January 2014

Academic Editor: Joohun Ha

Copyright (c) 2014 Mushtaq Ahmad Beigh et al. This is an open access article distributed under the Creative Commons Attribution License, which permits unrestricted use, distribution, and reproduction in any medium, provided the original work is properly cited.

\begin{abstract}
Bupivacaine is an amide type long acting local anesthetic used for epidural anesthesia and nerve blockade in patients. Use of bupivacaine is associated with severe cytotoxicity and apoptosis along with inhibition of cell growth and proliferation. Although inhibition of Erk, Akt, and AMPK seemingly appears to mediate some of the bupivacaine effects, potential downstream targets that mediate its effect remain unknown. S6 kinase 1 is a common downstream effector of several growth regulatory pathways involved in cell growth and proliferation known to be affected by bupivacaine. We have accordingly attempted to relate the growth inhibitory effects of bupivacaine with the status of S6K1 activity and we present evidence that decrease in cell growth and proliferation by bupivacaine is mediated through inactivation of S6 kinase 1 in a concentration and time dependent manner. We also show that ectopic expression of constitutively active S6 kinase 1 imparts substantial protection from bupivacaine induced cytotoxicity. Inactivation of S6K1 though associated with loss of putative mTOR mediated phosphorylation did not correspond with loss of similar phosphorylations in 4EBP1 indicating that S6K1 inhibition was not mediated through inactivation of mTORC1 signaling pathway or its down regulation.
\end{abstract}

\section{Introduction}

Bupivacaine is an amide type local anaesthetic used in clinical pain management [1]. Although considered safe, considerable side effects associated with its use remain a major concern. Bupivacaine though variable in efficacy inflicts myotoxicity and neurotoxicity and is also implicated in slowing down or preventing wound healing at surgical sites [2-6]. These effects have largely been attributed to its influence on cellular proliferation and cell death due to necrosis or apoptosis [7-9]. Accordingly studies have suggested that bupivacaine induced cell damage may involve participation of mitogen activated protein kinase (MAPK) and protein kinase B (Akt) signaling pathways [10-12]. Similarly disruption of other signalling events has been suggested for myotoxic effects associated with its use $[13,14]$. Since most of the pathways converge on S6 kinase, it was imperative to examine its relevance in mediating growth inhibitory effects associated with the drug.
Ribosomal protein S6 kinase 1 (S6K1) is an evolutionary conserved protein kinase that acts downstream of mTOR/PI3-kinase/Akt signalling pathway [15-18]. Growth factor dependent activation of this kinase is essential for the cell cycle progression primarily due to its influence on protein synthesis [19]. In addition, the role of S6K1 stands established in other cellular processes like autophagy, apoptosis, and aging implicating a complex network of signalling events in its regulation [20-22]. mTORC1 complex is considered to be the major activating input that regulates cellular growth through downstream effectors S6K1 and 4EBP1 (eukaryotic initiation factor 4E binding protein 1) [23] . Interaction between MAPK and S6K1 suggestive of cross talk between these two pathways stands well characterized, such that MAPK/Erk inhibitor, namely, PD-98059, inactivate S6K1 [24]. A recent observation by Choi et al. indicates that cellular death and survival pathways are regulated by Erk/GSK3 $\beta /$ S6K1 axis [25]. Similarly Akt-a downstream 


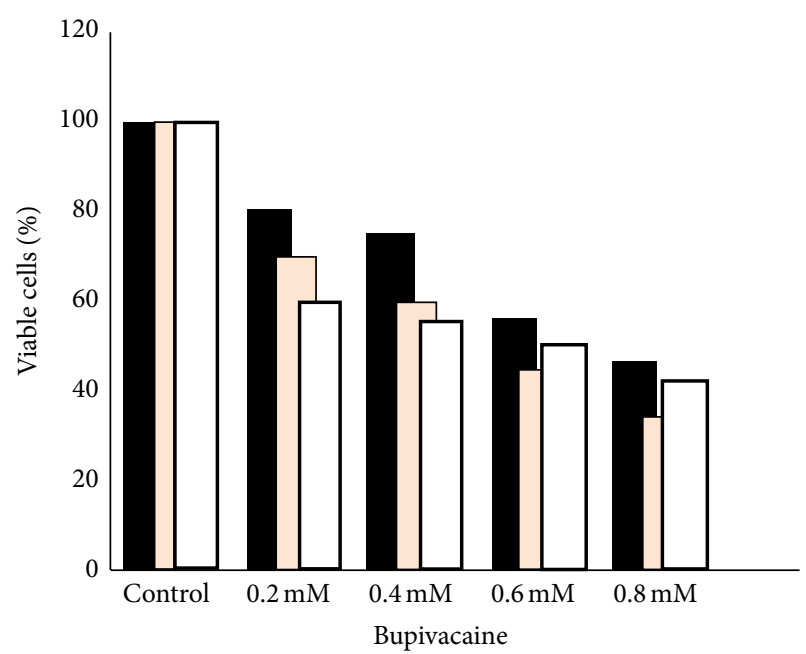

NIH-3T3 cells

C6 cells

$\mathrm{C} 2 \mathrm{C} 12$ cells

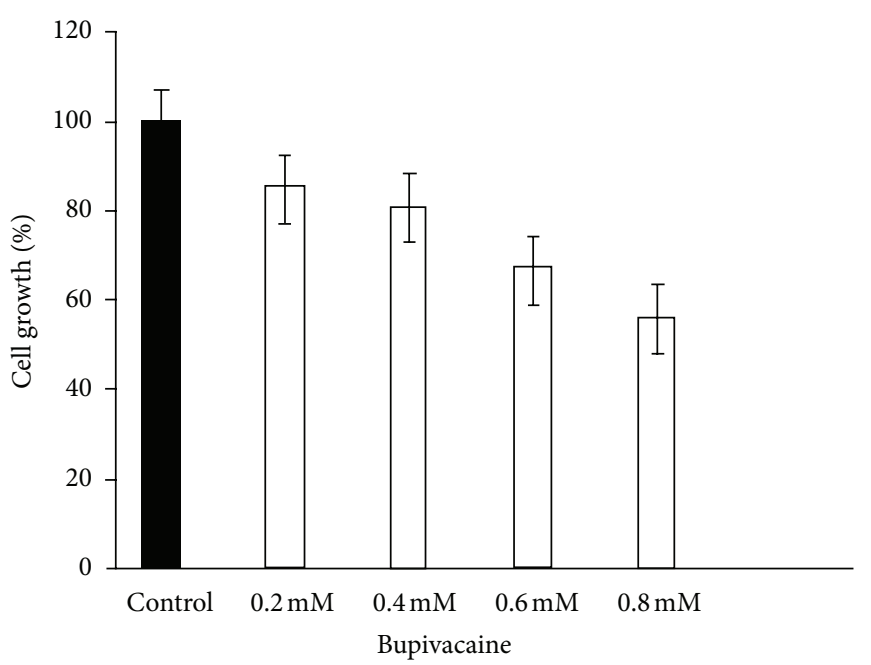

(b)

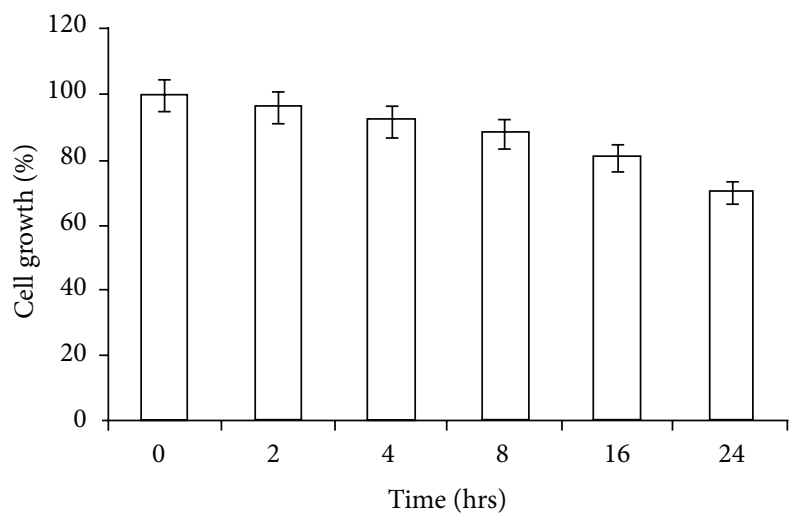

(c)

FIGURE 1: Dose-dependent inhibition of cell growth. (a) NIH-3T3, C2C12, and C6 neuroglial cells were plated at a cell density of $5 \times 10^{3}$ cells /well in triplicate under standard growth conditions (96-well format) for $24 \mathrm{hrs}$ and incubated with indicated concentrations of bupivacaine. Cells were washed with cold PBS and subjected to MTT assays. (b) Dose-dependent inhibition of cell growth in NIH-3T3 cells exposed to bupivacaine at indicated concentrations for $24 \mathrm{hrs}$. (c) Time dependent inhibition of cell growth in NIH cells treated with bupivacaine $(0.614 \mathrm{mM})$. Cell growth rate was normalized to the control. Data are mean $\pm \mathrm{SEM}(n=3) . P<0.05$ versus control.

effector of PI3-Kinase pathway is a known activator of S6K1 that caters to the response associated with insulin signalling [26].

Since the effects associated with bupivacaine are at least in part believed to be mediated by signals with potential to modulate S6K activity, we attempted to investigate such a possibility for further insights into the antiproliferative effects of the anaesthetic and relate it with inhibition of S6K pathway. Long established inhibitory influence of bupivacaine on amino acid sensing through tRNA charging invites further interest in potential involvement of S6 kinase pathway known to be critical for maintaining cellular nutrient homeostasis $[27,28]$. Inhibition of such signals is therefore likely to have a bearing on the activity status of the S6K1 enzyme.

\section{Materials and Methods}

2.1. Materials and Antibodies. Bupivacaine (Astra Zeneca), Protein G-Agarose beads (Calbiochem), DMEM, FBS, and antibiotics were purchased from Sigma-Aldrich; all other reagents were purchased from Sigma unless otherwise indicated. Anti-S6K antibody (Santa Cruz Biotechnologies, Inc.), Anti-S6K (Polyclonal-Genscript), Anti-Erk1, Antiphospho-Erk, Anti-4EBP1, Anti-phospho-4EBP1-T70 and Anti-phospho-S6K-T412 antibodies were obtained from Cell Signaling Technologies, Anti-phospho-S6K-T252 antibody was from R\&D systems, and Anti-PARP antibody was from Abcam, Inc. USA. PVDF membrane was from Millipore, Inc. and, goat anti-rabbit secondary antibody conjugated to IR Dye $800 \mathrm{CW}$ was from LI-COR Biotechnologies. 


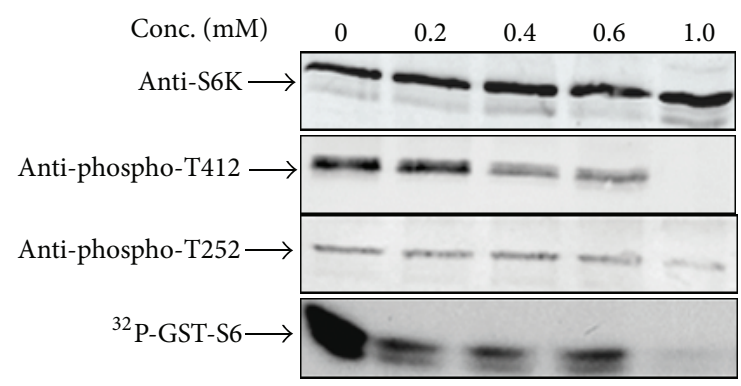

(a)

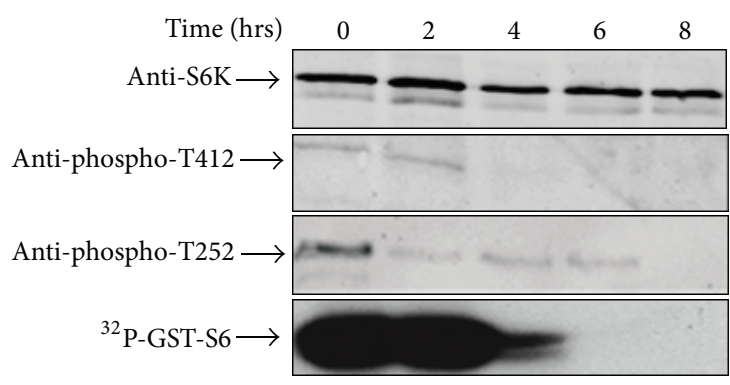

(c)

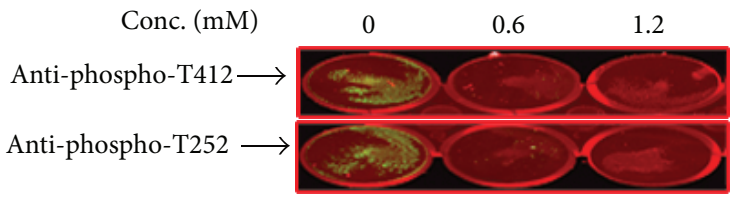

(e)

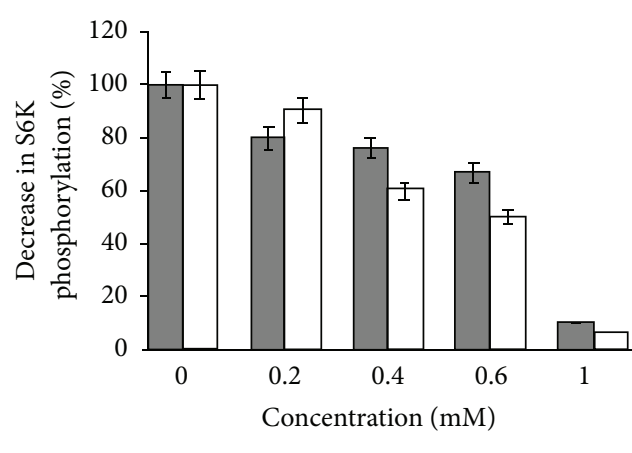

T412

ㅁ T252

(b)

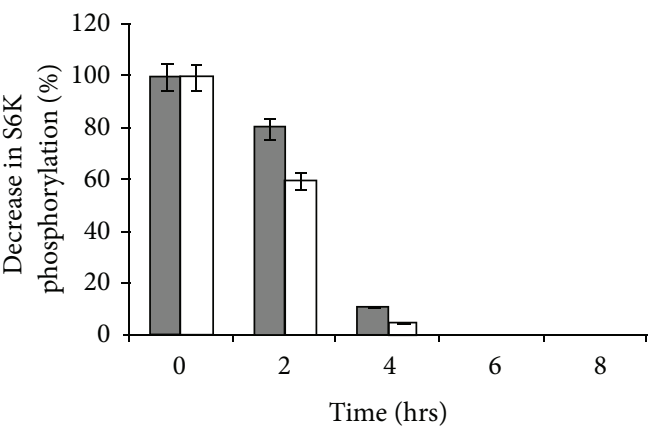

ㅁ T412

ㅁ T252

(d)

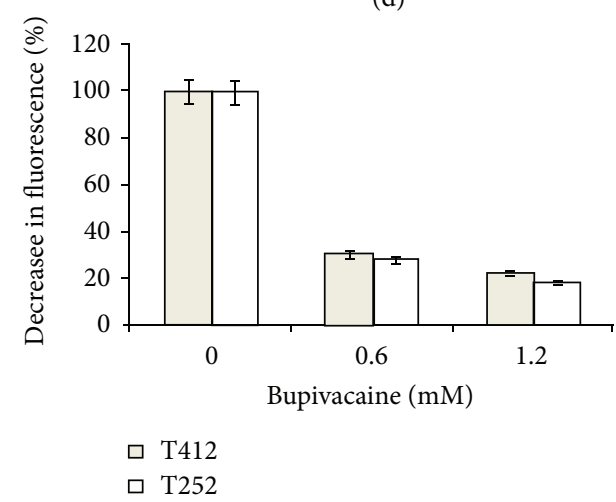

(f)

FIGURE 2: Concentration and time dependence of S6K1 inhibition by bupivacaine. (a) NIH-3T3 cells were allowed to grow for 12 hrs in the absence (control) or presence of indicated concentrations of bupivacaine (BPV) and S6K1 was immunoprecipitated, subjected to kinase assays, and then probed with indicated antibodies. (b) Densitometric analysis of anti-pS6K signals (normalized to total S6K levels) from three independent experiments. Data are relative S6K phosphorylation levels with control set as 100\%, presented as mean \pm SEM. (c) NIH$3 \mathrm{~T} 3$ cells were allowed to grow in the absence or presence of bupivacaine $(1 \mathrm{mM})$ for indicated time intervals and processed similarly as above. (d) Densitometric analysis of anti-pS6K signals (normalised to total S6K levels) from three independent experiments. (e) NIH-3T3 cells were incubated with inhibitory concentrations $(1 \mathrm{mM})$ of bupivacaine as described above. Cells were processed for treatment with S6K Phospho-T412 and S6K Phospho-T252 antibodies and imaged using LI-COR infrared imager. (f) Average florescent intensity of each well was calculated in arbitrary units (AU) using LI-COR ODYSSEY software.

2.2. Plasmid Constructs. HA (hemagglutinin) tagged S6 kinase, constitutively active HA-S6 kinase (S6K-T412E-DE3) and GST-S6 used as a substrate in S6K kinase assays were generated as described previously $[29,30]$.
2.3. Cell Culture and Transfections. NIH-3T3 fibroblasts, C2C12 myoblasts and C6 neuroglial cells were cultured in Dulbecco's modified Eagle's medium (with appropriate antibiotics) containing $10 \%(\mathrm{v} / \mathrm{v})$ fetal bovine serum in an 
atmosphere of $5 \% \mathrm{CO}_{2}$. Transfections were carried out using lipofectamine reagent (Invitrogen, Inc.) as per manufacturer's instructions in a 6-well plate format.

2.4. Drug Treatment, Immunoprecipitation, and Western Blotting. NIH-3T3 cells were incubated with indicated concentrations of drugs overnight or as shown. Cells were lysed in a buffer containing $50 \mathrm{mM}$ Tris- $\mathrm{HCl}$ (pH 7.5), $10 \mathrm{mM} \mathrm{MgCl}$, $5 \mathrm{mM}$ EDTA, $2 \mathrm{mM}$ DTT, $50 \mathrm{mM} \beta$-glycerophosphate, $0.5 \%$ Triton X-100 and protease inhibitor cocktail (Sigma) for 30 minutes and centrifuged at $15000 \mathrm{~g}$ for 30 minutes to clear the lysate. Clarified lysates were incubated with Anti-S6K antibody (mouse monoclonal immobilized on protein GAgarose beads) for 2-4 hrs. Beads were washed thrice with lysis buffer containing $500 \mathrm{mM} \mathrm{NaCl}$ before final washings with kinase buffer.

2.5. Immune Complex Kinase Assays. S6K1 immunoprecipitates were incubated with $1 \mu \mathrm{g}$ GST-S6 and $5 \mu \mathrm{Ci} \mathrm{p}{ }^{32}$-ATP in a kinase reaction buffer containing $50 \mathrm{mM}$ Tris- $\mathrm{Cl}(\mathrm{pH} \mathrm{7.0)}$, $10 \mathrm{mM} \mathrm{MgCl}_{2}, 0.5 \mathrm{mM}$ DTT, $50 \mathrm{mM} \beta$-glycerophosphate, and $1 \mathrm{mM}$ ATP for 20 minutes at $37^{\circ} \mathrm{C}$. Reaction was stopped by adding $2 \mathrm{X}$ loading buffer and separated on a 12\% SDSPAGE gel. Proteins were transferred on PVDF membrane, autoradiographed, and probed with S6K antibody (rabbit polyclonal) and other phospho antibodies followed by analysis with ODYSSEY infrared imager (LI-COR).

2.6. Proliferation Assays. Cells in triplicate (plated at density of 5000 cells per well) were grown in growth media in the presence or absence of bupivacaine for $24 \mathrm{hrs}$ or as indicated in 96-well format and subjected to MTT assays. The yellow tetrazolium salt MTT was reduced to formazan by intracellular NADPH-oxidoreductases. The formazan crystals were solubilized and quantified by spectrophotometry using plate reader (Epoch Biotech, USA). Assays were performed using an MTT cell proliferation kit (Sigma Aldrich, USA).

\subsection{In-Cell Western Assays and Fluorescence Quantitation.} In-cell western assays were done according to the protocol provided by LI-COR Biotechnologies. Briefly, NIH-3T3 cells seeded in 12-well plates were treated with S6K1 inhibitory concentrations of bupivacaine overnight. For analysis, cells were washed with PBS, fixed using 4\% paraformaldehyde, permeabilized using $0.1 \%$ Triton X-100 in PBS, blocked, and then incubated with Anti-phospho-S6K antibodies in LICOR blocking reagent overnight and average florescence intensity (in arbitrary units) of each well was quantitated using ODYSSEY infrared imager (LI-COR Biotechnologies).

2.8. Cell Death Assay. ELISA based cell death detection kit (Roche Applied Sciences) was used to detect apoptosis after bupivacaine treatment. The assay is based on a quantitative sandwich ELISA using antibodies directed against DNA and histones to detect mono- and oligonucleosomes in the cytoplasm of cells undergoing apoptosis. ELISA was carried out according to the manufacturer's protocol. Measured OD was normalized for cell number (protein content).

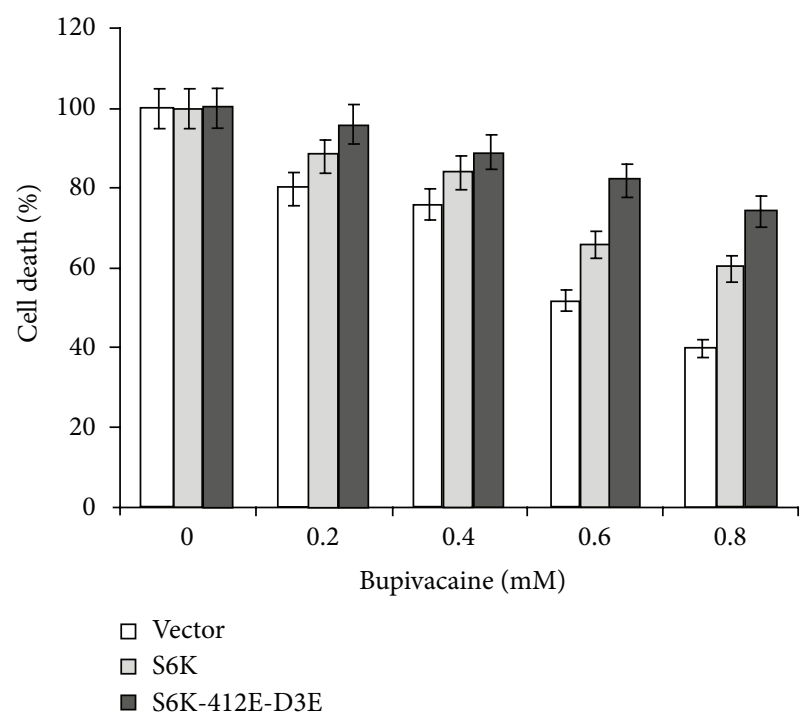

FIGURE 3: Constitutively active S6 kinase prevents cells from bupivacaine induced toxicity: NIH-3T3 cells transfected with HA-S6K1 and constitutively active S6K (S6K-T412E-D3E). 48 hrs posttransfection cells were exposed to various concentrations of bupivacaine $(1 \mathrm{mM})$ for $12 \mathrm{hrs}$ before harvest. Data are representative of three independent experiments.

2.9. Statistical Analysis and Calculation of IC50. Representative experiments from three independent experiments are shown. Results for each experiment are given as mean of triplicates \pm SD. Statistically significant differences between samples are determined using Student's t-tests (Excel, Microsoft). The half maximal inhibitory concentration (IC50) of bupivacaine was determined by nonlinear least-squares fitting of the data of MTT assays using the following equation: Normalized Cell Number $=1 /(1+[\mathrm{BPV}] / \mathrm{IC50})$, with Graph Pad Prism Software (Version 4.0).

\section{Results}

3.1. Bupivacaine Induces Toxicity in a Cell Type Specific and Dose-Dependent Manner. Cytotoxicity of bupivacaine was evaluated by its effect on cell viability. Accordingly cell lines $\mathrm{NIH}-3 \mathrm{~T} 3, \mathrm{C} 6$, and $\mathrm{C} 2 \mathrm{C} 12$ myoblasts were allowed to grow in the absence (control) or presence of bupivacaine at various concentrations over a period of $24 \mathrm{hrs}$. While the control cells exhibited normal growth characteristics, significant dose-dependent decline in cellular density was observed in bupivacaine treated cells resulting in substantial cell death (Figure 1(a)). The magnitude of cell death, however, varied with individual cell type such that maximum sensitivity to bupivacaine induced toxicity was most pronounced for C6 neurological cells recording more than 60\% death at concentrations $(0.2 \mathrm{mM})$ which did not cause substantial decline in densities of other cell types. While an intermediate toxicity response was observed for $\mathrm{C} 2 \mathrm{C} 12$ cells, the $\mathrm{NIH}-3 \mathrm{~T} 3$ cells were the least sensitive with an IC50 of $0.614 \mathrm{mM}$ which were accordingly deemed appropriate for reliable assessment of signaling dynamics associated with bupivacaine induced 


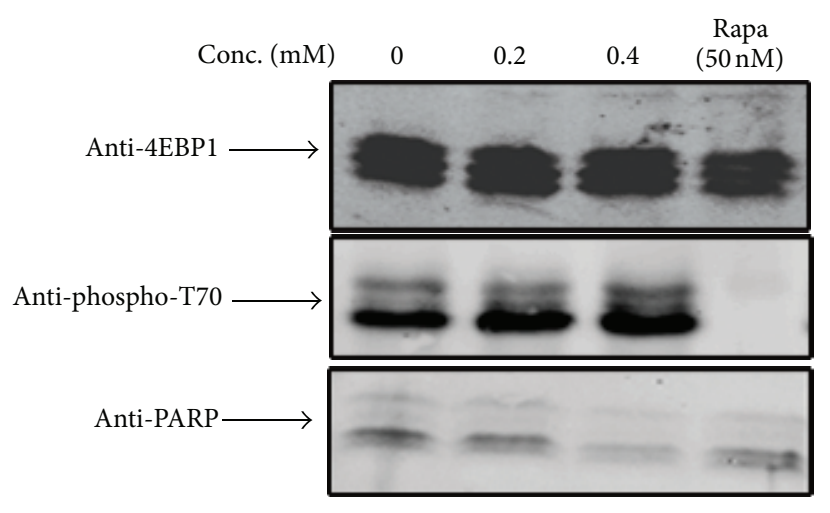

(a)

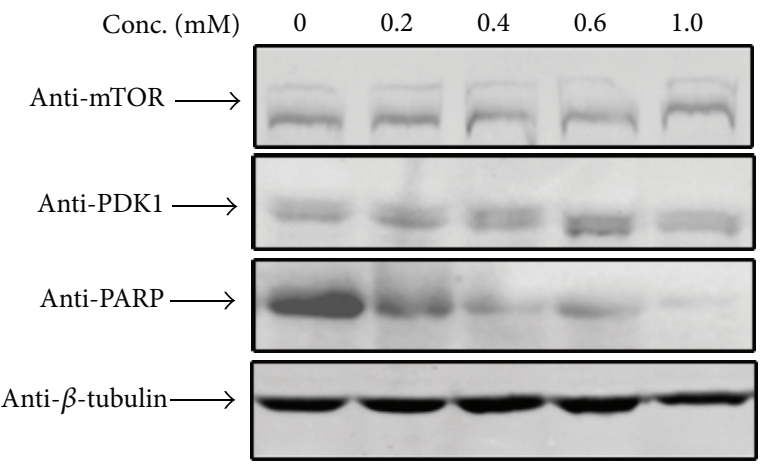

(b)

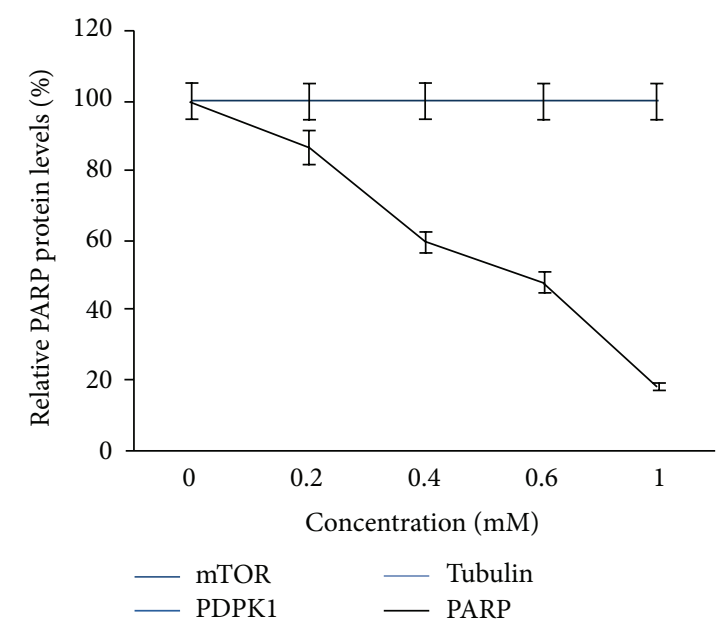

(c)

FIGURE 4: BPV mediated inhibition is independent of mTOR input: (a) NIH-3T3 cells were exposed to various concentrations of bupivacaine and incubated for $12 \mathrm{hrs}$ before harvest. 4EBP1 was immunoprecipitated with monoclonal antibody cross-linked to protein G-Agarose beads and immune complexes recovered were separated on a 15\% SDS-PAGE gel, transferred on PVDF membrane, and immunoblotted with rabbit anti-4EBP1 and anti-phospho-T70; Rapamycin was used as a positive control to inhibit mTORC1 complex (lane 4). (b) NIH-3T3 cells were grown in the absence (control) or presence of bupivacaine $(1 \mathrm{mM})$ for $12 \mathrm{~h}$ and subjected to immunoblotting with anti-mTOR, anti-PDPK1, anti-PARP, and anti $\beta$-tubulin antibodies, respectively, to detect endogenous levels of these proteins. (c) Expression analysis of $\beta$-tubulin relative to PARP protein levels (positive control) using fluorescence based quantitation by LI-COR-ODYSSEY software. Data are mean of three independent experiments.

toxicity without undue interference from cessation of cellular processes. NIH-3T3 cells were used further for studying their behavior in response to bupivacaine treatment and loss of cell viability potentiated by morphological changes like cell rounding and detachment was associated with a dosedependent decrease in cellular proliferation as assessed by MTT assay (Figure 1(b)). A time dependent assay in NIH cells with minimal inhibitory concentration $(0.614 \mathrm{mM})$ showed cells to be more than $70 \%$ viable at 24 hrs (Figure 1(c)).

\subsection{Bupivacaine Inhibits S6K1 in a Concentration and Time} Dependent Manner. Growth inhibition and apoptosis have quite often been associated with dysregulation of signaling pathways with potential to influence S6K1 activity directly or indirectly. We therefore, sought to investigate any such possibility by analyzing activity status of S6K1 in the presence or the absence of the drug. Endogenous S6K1 was immunoprecipitated from NIH-3T3 cells grown in presence or absence of different bupivacaine concentrations for its ability to phosphorylate GST-S6. As seen in Figure 2(a), bupivacaine caused $\mathrm{S} 6 \mathrm{~K} 1$ inhibition in a concentration dependent manner with its near complete inhibition at a drug concentration of $1 \pm 0.06 \mathrm{mM}$. Minimal inhibitory concentration for $\mathrm{S} 6 \mathrm{~K} 1$ was then used to establish the time course of S6K1 inhibition. Figure 2(c) shows that inhibition of S6K1 activity was stringently time dependent with more than $80 \%$ inhibition observed at 4 hours of the drug exposure. The inhibitory time course was in concordance with inhibition of cell proliferation.

3.3. S6K1 Inhibition Is Associated with Loss of Activating Phosphorylations. Catalytic and linker domain phosphorylations at the activation loop (AL) and hydrophobic motifs (HM) are established determinants of S6K1 enzyme activity. 
Accordingly their loss is a hallmark of S6K1 inhibition. We therefore sought to ascertain whether inhibition of S6K1 by bupivacaine did indeed correspond with loss of these phosphorylations. As seen in Figure 2(a), both T412 and T252 phosphorylations were lost in a concentration dependent manner which corresponded with activity profile of the enzyme to exhibit a 20-90\% drop in fluorescent intensity over the entire range of drug concentrations tested (Figure 2(b)). The loss of both the phosphorylations followed a strict time course with $90 \%$ loss of both the phosphorylations at $4 \mathrm{~h}$ (Figure 2(d)). In order to rule out that the observed loss of S6K1 phosphorylations was not due to a contaminating phosphatase, an in-cell western assay in NIH-3T3 cells using phospho specific antibodies against Threonine 412 and Threonine 252 in S6 kinase was performed in the presence or absence of the drug. As seen in Figure 2(e), the relative fluorescent intensity for the individual phospho-antibodies exhibited similar pattern of disappearance as observed for the immunoprecipitated enzyme. At bupivacaine concentration of $1.2 \mathrm{mM}$ the relative phosphorylation decreased to $20 \%$ when quantitated using LI-COR infrared imager (Figure 2(f)).

3.4. Constitutive Active S6K1 Induces Resistance to Bupivacaine Toxicity. In an attempt to establish that $\mathrm{S} 6 \mathrm{~K} 1$ inactivation did indeed mediate the growth inhibitory effects associated with bupivacaine, cells transfected with HA-tagged S6K1 wild type and a constitutively active variant T412E-D3E were examined for their ability to resist bupivacaine induced toxicity. Figure 3 shows that while WT S6K1 was able to significantly improve the live cell number, it remained partially protective $(30 \%)$ in comparison to a dramatic $80-85 \%$ protection conferred in cells expressing the constitutive active variant of the enzyme. These data clearly indicate that S6K1 did indeed mediate growth inhibition by bupivacaine.

3.5. S6K1 Inhibition by Bupivacaine Is mTORC1 Independent. mTORC1 is considered to be a major input acting upstream of S6K1 and implicated in T412 phosphorylation of the enzyme and parallel phosphorylations of its other substrate, eukaryotic translation initiation factor $4 \mathrm{E}$ binding protein 1 (4EBP1). Possible inactivation of mTOR pathway suggested by inhibition of S6K1 should therefore be corroborated by its inability to cause phosphorylations in 4EBP1 (Threonine 70). Phosphorylation of 4EBP1 at Threonine 70 site (Figure 4(a)) remained unchanged in the presence of bupivacaine when compared to its inhibition by specific mTORC1 inhibitor rapamycin. To completely rule out any possible mTORC1 input for $\mathrm{S} 6 \mathrm{~K}$ inhibition mediated by bupivacaine, an immunoblot analysis of endogenous mTOR and PDPK1 (Figure 4(b)) indicated that the drug did not cause any significant change in the expression of either kinase implicated in phosphorylation at HM (T412) and AL (T252), respectively, when compared to PARP (poly-ADP ribose polymerase) that recorded visible decrease in protein levels (Figure 4(c)) in conformity with its reported response to bupivacaine [31]. This goes to suggest that the selective

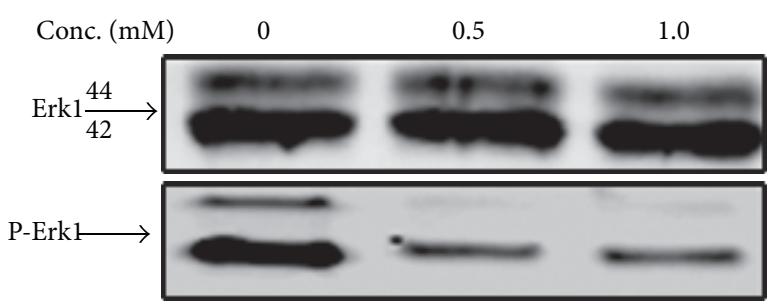

(a)

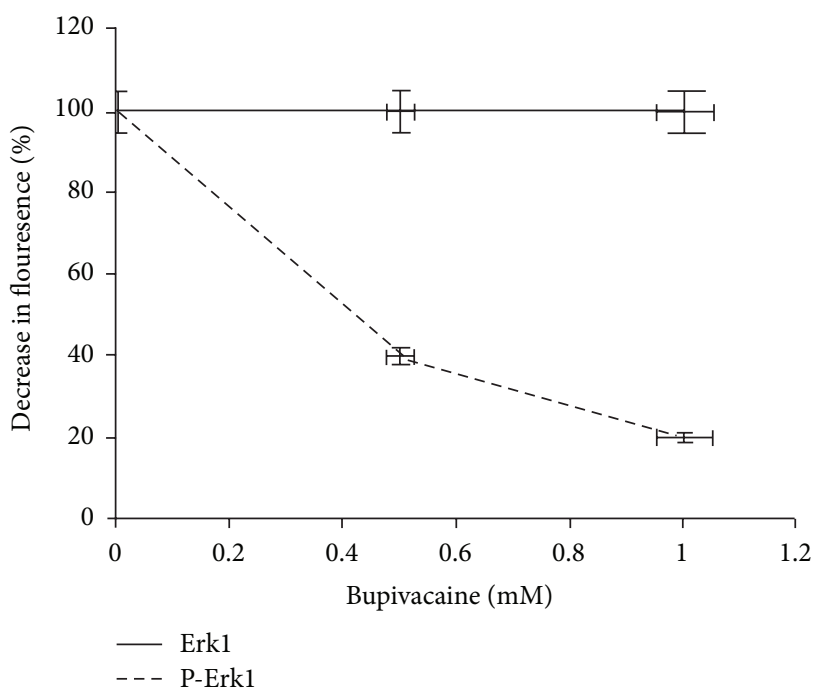

(b)

FIGURE 5: Overlapping time course of inhibition between Erk and S6K1 indicates bupivacaine as specific inhibitor of Erk and S6K1: (a) NIH-3T3 cells treated with indicated concentrations of bupivacaine as above. Lysates were clarified, immunoprecipitated, and probed with indicated antibodies. (b) Densitometric analysis of anti-P-Erk1 signals (normalized to total Erk levels) from three independent experiments. Data are relative Erk activation levels with control set as $100 \%$, presented as mean \pm SEM.

inhibition of $\mathrm{S} 6 \mathrm{~K} 1$ was in no way related to change in activity or expression characteristics of mTOR or PDPK1.

3.6. S6K1 Inhibition by Bupivacaine Overlaps with Erk Inactivation. In the absence of mTOR as the mediator of S6K1 inhibition by bupivacaine, it was imperative to examine other signaling pathways that may directly or indirectly influence S6K1 activity in the cell. Since extracellular signal-related kinase/s (Erk) are believed to participate in the events leading to cytotoxicity by anesthetic drugs like bupivacaine, we sought to investigate the activation status of Erks to possibly relate it with observed inhibition of cellular proliferation and S6K1 activity. The activation of Erks was evaluated by immunoblotting with a phospho specific antibody. As seen in Figure 5(a), bupivacaine caused a dramatic reduction in Erk phosphorylation in a dose-dependent manner. Densitometric analysis (Figure 5(b)) of the data from several immunoblots $(n=3)$ indicated that the extent of Erk inhibition coincided with the inhibition of S6K1 as much as it did with the kinetics of cell proliferation, to suggest that the 
effect of bupivacaine may be mediated through inactivation of Erk and S6K1.

\section{Discussion}

The use of local anesthetics like bupivacaine is associated with chondrotoxicity, myotoxicity, and neurotoxicity at variable propensities $[4,9,32]$. Diverse spectrums of cellular changes that underline dysfunctional signal transduction are attributed to mediate cellular damage associated with their use [32]. Inhibition of cell growth, a key effect associated with the use of these drugs particularly bupivacaine, has invited significant interest leading to the identification of a series of signaling molecules that appear to mediate the process [11, 32]. Since growth regulatory and apoptotic mechanisms are largely interdependent and complexed further by cross talk among signaling pathways, the attribution of one or the other event to mediate such effects may be premature. Further, the influence of the other signaling pathways like S6K1 on cellular growth and apoptosis is as pronounced as, if not more than, the events reported to get influenced by such drugs $[8,10,20]$. Incidentally the effects associated with bupivacaine that include metabolic stress, influence on nutrient homeostasis, and apoptosis directly or indirectly suggest a possible involvement of S6K pathway, to rationalize this study.

We chose NIH-3T3 cells for their restrained response to bupivacaine induced cytotoxicity compared to C2C12 and C6 cell lines, to help us register the dynamics of signaling events responsible for preventing cell growth and proliferation. We demonstrate that the inhibition of cell proliferation by bupivacaine strictly correlated with the inactivation of ribosomal protein S6 kinase 1 and its relief by the use of constitutively active enzyme to suggest a possible dysregulation of events that control cell division, at least in part via protein translation. With long standing implications that bupivacaine may influence protein synthesis by interfering with amino acylation of tRNAs [28], the relevance of S6K1 inhibition becomes even more compelling. Therefore, evaluating the status of different signaling molecules known to influence S6K1 activity directly or indirectly becomes imperative to establish their relation with bupivacaine action. The principal regulatory input for $\mathrm{S} 6 \mathrm{~K} 1$ is believed to be provided by mTOR signaling pathway wherein mTOR kinase directly phosphorylates $\mathrm{S} 6 \mathrm{~K} 1$ at its hydrophobic motif to trigger a second phosphorylation at its activation loop for full activation [27, 33]. Sequential dynamics of the two phosphorylations is, therefore, an established index of S6K1 activity and often related to the activity status of the mTOR pathway per se. However, DRAK2, Akt, GSK3, and Erk like kinases believed to phosphorylate putative mTOR site in S6K1 rope in other signaling pathways as possible regulators of S6K1 [17, 24, $34,35]$. Bupivacaine induced loss of S6K1 phosphorylation at putative mTOR site and at the activation loop as a consequence, without any perceptible change in mTOR kinase activity or expression observed herein, substantiates the later possibility even further. Incidentally, all these kinases have established influence in the process of cell growth and apoptosis and each would easily fit into a role of mediating growth inhibition by bupivacaine, with a marginal preference at best, for one over the other. Despite its established role in cellular apoptosis, DRAK2 may slip down the preference list for its immune cell specific response, yet its participation in the mechanistic events associated with bupivacaine cannot be ruled out completely. On the other hand, Akt presents credible candidature in light of its established role in S6K regulation coupled with its documented inhibition by bupivacaine $[11,26]$. Since Akt influence on S6K1 remains largely dependent on its cross talk with mTOR pathway [36], its exclusive participation in mediating growth inhibition by bupivacaine becomes difficult to explain. That is perhaps why Akt has often been implicated in mediating apoptosis, an event that comes next to growth inhibition. Our observation that Akt phosphorylation by bupivacaine was inhibited much later in time and at concentrations higher than required for S6K inhibition (data not shown) may support the contention further. However, involvement of other signaling pathways that may recruit Akt to mediate other effects associated with bupivacaine cannot be ruled out. This is particularly relevant in the context of the data that p38MAPK and c-Jun Nterminal kinase pathways stand implicated in bupivacaine induced neurotoxicity $[10,11,32]$, whereas mitochondrial dysfunction and altered calcium homeostasis are suggested to mediate myotoxicity $[14,37]$. These observations indicate the complex nature of events that may collaborate to bring about cumulative effects associated with bupivacaine or else the drug may engage distinct signaling pathways in a function specific and/or a cell specific manner.

MAPK signaling has a central role in regulating cell growth and proliferation [38]. Accordingly cell division is facilitated by activation of Erks and prevented by agents with the ability to inhibit their activation $[39,40]$. The contribution of $\mathrm{S} 6 \mathrm{~K} 1$ in promoting cell division is no less pronounced and equates well with that of Erks especially in the context of its inhibition by bupivacaine with overlapping dose and time kinetics. The observation lends support to the contention of a cross talk between Erk and S6K1 pathways, in agreement with the data describing MAPK-S6K1 coimmunoprecipitation to suggest a coordinated Erk-GSK3B-S6K1 axis for cell growth and proliferation $[12,24,34]$. Incidentally both Erk and GSK3 are known to influence phosphorylation status of S6K1 to contribute in direct activation of S6K1 [34]. Accordingly agents that inhibit upstream members of the MAPK pathway resulting in ERK inactivation also seem to influence the activity status of S6K1. It is, however, premature to attribute a general role for Erks in $\mathrm{S6K1}$ activation in preference to mTOR and it should instead be considered a consequence associated with the use of bupivacaine. Paradoxically, the contention that mTOR regulates $\mathrm{S} 6 \mathrm{~K} 1$ by a mechanism other than phosphorylation [30] may implicate Erks in a more general role.

\section{Conclusions}

Our data suggests that bupivacaine induced inhibition of cell proliferation is mediated at least in part through inactivation of S6 kinase 1. We also demonstrate redundant role of mTORC1 signaling in mediating this effect to suggest the possible involvement of Erk pathway. 


\section{Conflict of Interests}

The authors declare no financial or nonfinancial conflict of interests.

\section{Authors' Contribution}

Khurshid Iqbal Andrabi and Mushtaq Ahmad Beigh conceived and designed the experiments. Mushtaq Ahmad Beigh and Mehvish Showkat performed the experiments. Mushtaq Ahmad Beigh, Mehvish Showkat, and Khurshid Iqbal Andrabi analyzed the data. Mehvish Showkat, Basharat Bashir, and Asma Bashir contributed reagents/materials/ analysis tools. Mushtaq Ahmad Beigh, Mehvish Showkat, and Khurshid Iqbal Andrabi wrote the paper.

\section{Acknowledgments}

The authors greatly appreciate the kind gift of HA-S6K $\alpha 1$ in PMT2 vector from Dr. Joseph Avruch, Mass. General Hospital, Harvard Medical School, Boston, MA. Junior Research Fellowships in favour of Mushtaq Ahmad Beigh (09/251/0025) and Mehvish Showkat (20-6/2009) from the Council of Scientific \& Industrial Research (CSIR) and University Grants Commission (UGC), respectively, are great fully acknowledged. Infrastructural Grant under FIST program and Special Assistance Grant under SAP program from DST and UGC are duly acknowledged.

\section{References}

[1] N. Malhotra, C. Chanana, K. K. Roy, S. Kumar, V. Rewari, and J. B. Sharma, "To compare the efficacy of two doses of intraperitoneal bupivacaine for pain relief after operative laparoscopy in gynecology," Archives of Gynecology and Obstetrics, vol. 276, no. 4, pp. 323-326, 2007.

[2] S. Y. Fong, T. J. Pavy, S. T. Yeo, M. J. Paech, and L. C. Gurrin, "Assessment of wound infiltration with bupivacaine in women undergoing day-case gynecological laparoscopy," Regional Anesthesia and Pain Medicine, vol. 26, no. 2, pp. 131136, 2001.

[3] J. I. Einarsson, J. Sun, J. Orav, and A. E. Young, "Local analgesia in laparoscopy: a randomized trial," Obstetrics and Gynecology, vol. 104, no. 6, pp. 1335-1339, 2004.

[4] A. H. Foster and B. M. Carlson, "Myotoxicity of local anesthetics and regeneration of the damaged muscle fibers," Anesthesia and Analgesia, vol. 59, no. 10, pp. 727-736, 1980.

[5] W. Zink, C. Seif, J. R. E. Bohl et al., "The acute myotoxic effects of bupivacaine and ropivacaine after continuous peripheral nerve blockades," Anesthesia and Analgesia, vol. 97, no. 4, pp. 11731179, 2003.

[6] I. A. M. Radwan, S. Saito, and F. Goto, "The neurotoxicity of local anesthetics on growing neurons: a comparative study of lidocaine, bupivacaine, mepivacaine, and ropivacaine," Anesthesia and Analgesia, vol. 94, no. 2, pp. 319-324, 2002.

[7] E. Boselli, F. Duflo, R. Debon et al., "The induction of apoptosis by local anesthetics: a comparison between lidocaine and ropivacaine," Anesthesia and Analgesia, vol. 96, no. 3, pp. 755756, 2003.
[8] C. J. Park, S. A. Park, T. G. Yoon, S. J. Lee, K. W. Yum, and H. J. Kim, "Bupivacaine induces apoptosis via ROS in the Schwann cell line," Journal of Dental Research, vol. 84, no. 9, pp. 852-857, 2005.

[9] C. R. Chu, N. J. Izzo, C. H. Coyle, N. E. Papas, and A. Logar, "The in vitro effects of bupivacaine on articular chondrocytes," Journal of Bone and Joint Surgery B, vol. 90, no. 6, pp. 814-820, 2008.

[10] P. Lirk, I. Haller, H. Peter et al., "In vitro, inhibition of mitogenactivated protein kinase pathways protects against bupivacaineand ropivacaine-induced neurotoxicity," Anesthesia and Analgesia, vol. 106, no. 5, pp. 1456-1464, 2008.

[11] J. M. Maurice, Y. Gan, F.-X. Ma, Y.-C. Chang, M. Hibner, and Y. Huang, "Bupivacaine causes cytotoxicity in mouse C2C12 myoblast cells: involvement of ERK and Akt signaling pathways," Acta Pharmacologica Sinica, vol. 31, no. 4, pp. 493500, 2010.

[12] Z. Wang, J. Shen, J. Wang et al., "Lithium attenuates bupivacaine-induced neurotoxicity in vitro through phosphatidylinositol-3-kinase/threonine-serine protein kinase B- and extracellular signal-regulated kinase-dependent mechanisms," Neuroscience, vol. 206, pp. 190-200, 2012.

[13] Y. Huang, J. Li, Y. Zhang, and C. Wu, "The roles of integrinlinked kinase in the regulation of myogenic differentiation," The Journal of Cell Biology, vol. 150, no. 4, pp. 861-872, 2000.

[14] W. Irwin, E. Fontaine, L. Agnolucci et al., "Bupivacaine myotoxicity is mediated by mitochondria," Journal of Biological Chemistry, vol. 277, no. 14, pp. 12221-12227, 2002.

[15] D. Alessi, M. T. Kozlowski, Q.-P. Weng, N. Morrice, and J. Avruch, "3-phosphoinositide-dependent protein kinase 1 (PDK1) phosphorylates and activates the p70 S6 kinase in vivo and in vitro," Current Biology, vol. 8, no. 2, pp. 69-81, 1998.

[16] D. J. Price, J. Russell Grove, V. Calvo, J. Avruch, and B. E. Bierer, "Rapamycin-induced inhibition of the 70-kilodalton S6 protein kinase," Science, vol. 257, no. 5072, pp. 973-977, 1992.

[17] M. J. Riemenschneider, R. A. Betensky, S. M. Pasedag, and D. N. Louis, "AKT activation in human glioblastomas enhances proliferation via TSC2 and S6 kinase signaling," Cancer Research, vol. 66, no. 11, pp. 5618-5623, 2006.

[18] S. Isotani, K. Hara, C. Tokunaga, H. Inoue, J. Avruch, and K. Yonezawa, "Immunopurified mammalian target of rapamycin phosphorylates and activates p70 S6 kinase a in vitro," Journal of Biological Chemistry, vol. 274, no. 48, pp. 34493-34498, 1999.

[19] K. A. Martin and J. Blenis, "Coordinate regulation of translation by the PI 3-kinase and mTOR pathways," Advances in Cancer Research, vol. 86, pp. 1-39, 2002.

[20] H. Harada, J. S. Andersen, M. Mann, N. Terada, and S. J. Korsmeyer, "p70S6 kinase signals cell survival as well as growth, inactivating the pro-apoptotic molecule BAD," Proceedings of the National Academy of Sciences of the United States of America, vol. 98, no. 17, pp. 9666-9670, 2001.

[21] S. M. Armour, J. A. Baur, S. N. Hsieh, A. Land-Bracha, S. M. Thomas, and D. A. Sinclair, "Inhibition of mammalian S6 kinase by resveratrol suppresses autophagy," Aging, vol.1, no. 6, pp. 515528, 2009.

[22] S. H. Um, F. Frigerio, M. Watanabe et al., "Absence of S6K1 protects against age- and diet-induced obesity while enhancing insulin sensitivity," Nature, vol. 431, no. 7005, pp. 200-205, 2004.

[23] H. Nojima, C. Tokunaga, S. Eguchi et al., "The Mammalian Target Of Rapamycin (mTOR) partner, raptor, binds the mTOR substrates p70 S6 kinase and 4E-BP1 through their TOR 
signaling (TOS) motif," Journal of Biological Chemistry, vol. 278, no. 18, pp. 15461-15464, 2003.

[24] J. A. Lehman and J. Gomez-Cambronero, "Molecular crosstalk between p70S6k and MAPK cell signaling pathways," Biochemical and Biophysical Research Communications, vol. 293, no. 1, pp. 463-469, 2002.

[25] C.-H. Choi, B.-H. Lee, S.-G. Ahn, and S.-H. Oh, "Proteasome inhibition-induced p38 MAPK/ERK signaling regulates autophagy and apoptosis through the dual phosphorylation of glycogen synthase kinase $3 \beta$," Biochemical and Biophysical Research Communications, vol. 418, no. 4, pp. 759-764, 2012.

[26] J. M. Lizcano, S. Alrubaie, A. Kieloch, M. Deak, S. J. Leevers, and D. R. Alessi, "Insulin-induced Drosophila S6 kinase activation requires phosphoinositide 3-kinase and protein kinase B," Biochemical Journal, vol. 374, no. 2, pp. 297-306, 2003.

[27] X. Wang, L. E. Campbell, C. M. Miller, and C. G. Proud, "Amino acid availability regulates p70 S6 kinase and multiple translation factors," Biochemical Journal, vol. 334, no. 1, pp. 261-267, 1998.

[28] M. E. Johnson and G. H. Jones, "Effects of marcaine, a myotoxic drug, on macromolecular synthesis in muscle," Biochemical Pharmacology, vol. 27, no. 13, pp. 1753-1757, 1978.

[29] M. A. Beigh, M. Showkat, M. U. Hussain, and K. I. Andrabi, "Loss of hydrophobic motif and activation loop phosphorylation is a consequence and not the mechanism of S6 kinase 1 inhibition by rapamycin," Journal of Biological Regulators and Homeostatic Agents, vol. 27, no. 2, pp. 399-408, 2013.

[30] M. A. Beigh, M. Showkat, M. Ul Hussain, S. A. Latoo, S. T. Majeed, and K. I. Andrabi, "Rapamycin inhibition of Baculovirus Recombinant (BVr) ribosomal protein S6 Kinase (S6K1) is mediated by an event other than phosphorylation," Cell Commun Signal, vol. 10, article 4, 2012.

[31] A. Unami, Y. Shinohara, T. Ichikawa, and Y. Baba, "Biochemical and microarray analyses of bupivacaine-induced apoptosis," The Journal of Toxicological Sciences, vol. 28, no. 2, pp. 77-94, 2003.

[32] F. Yanagidate and G. R. Strichartz, "Bupivacaine inhibits activation of neuronal spinal extracellular receptor-activated kinase through selective effects on ionotropic receptors," Anesthesiology, vol. 104, no. 4, pp. 805-814, 2006.

[33] K. Hara, K. Yonezawa, Q.-P. Weng, M. T. Kozlowski, C. Belham, and J. Avruch, "Amino acid sufficiency and mTOR regulate p70 S6 kinase and eIF-4E BP1 through a common effector mechanism," Journal of Biological Chemistry, vol. 273, no. 23, pp. 14484-14494, 1998.

[34] S. Shin, L. Wolgamott, Y. Yu, J. Blenis, and S.-O. Yoon, "Glycogen Synthase Kinase (GSK)-3 promotes p70 ribosomal protein S6 kinase (p70S6K) activity and cell proliferation," Proceedings of the National Academy of Sciences of the United States of America, vol. 108, no. 47, pp. E1204-E1213, 2011.

[35] J. Mao, H. Luo, B. Han, R. Bertrand, and J. Wu, "Drak2 is upstream of p70S6 kinase: its implication in cytokine-induced islet apoptosis, diabetes, and islet transplantation," Journal of Immunology, vol. 182, no. 8, pp. 4762-4770, 2009.

[36] A. L. Edinger and C. B. Thompson, "Akt maintains cell size and survival by increasing mTOR-dependent nutrient uptake," Molecular Biology of the Cell, vol. 13, no. 7, pp. 2276-2288, 2002.

[37] P. Partownavid, S. Umar, J. Li, S. Rahman, and M. Eghbali, "Fatty-acid oxidation and calcium homeostasis are involved in the rescue of bupivacaine-induced cardiotoxicity by lipid emulsion in rats," Critical Care Medicine, vol. 40, no. 8, pp. 24312437, 2012.
[38] M. Meister, A. Tomasovic, A. Banning, and R. Tikkanen, "Mitogen-Activated Protein (MAP) kinase scaffolding proteins: a recount," International Journal of Molecular Sciences, vol. 14, no. 3, pp. 4854-4884, 2013.

[39] T.-Y. Huang, W.-C. Chang, M.-Y. Wang, Y.-R. Yang, and Y.-C. Hsu, "Effect of sulforaphane on growth inhibition in human brain malignant glioma GBM 8401 cells by means of mitochondrial- and MEK/ERK-mediated apoptosis pathway," Cell Biochemistry and Biophysics, pp. 1-13, 2012.

[40] E. J. Park, H. J. Chung, H. J. Park, G. D. Kim, Y. H. Ahn, and S. K. Lee, "Suppression of Src/ERK and GSK-3/beta-catenin signaling by pinosylvin inhibits the growth of human colorectal cancer cells," Food and Chemical Toxicology, vol. 55, pp. 424433, 2013. 

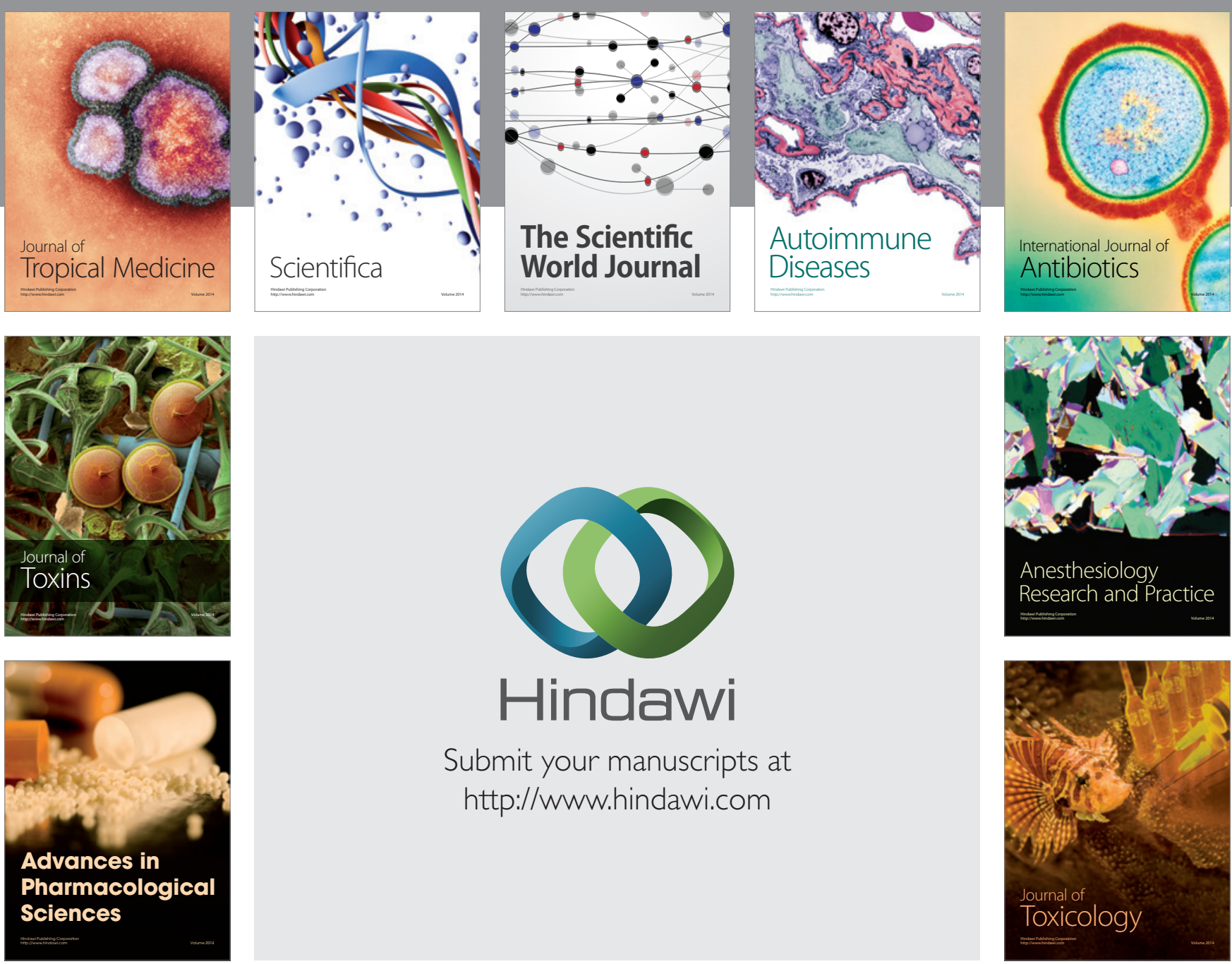

\section{Hindawi}

Submit your manuscripts at

http://www.hindawi.com
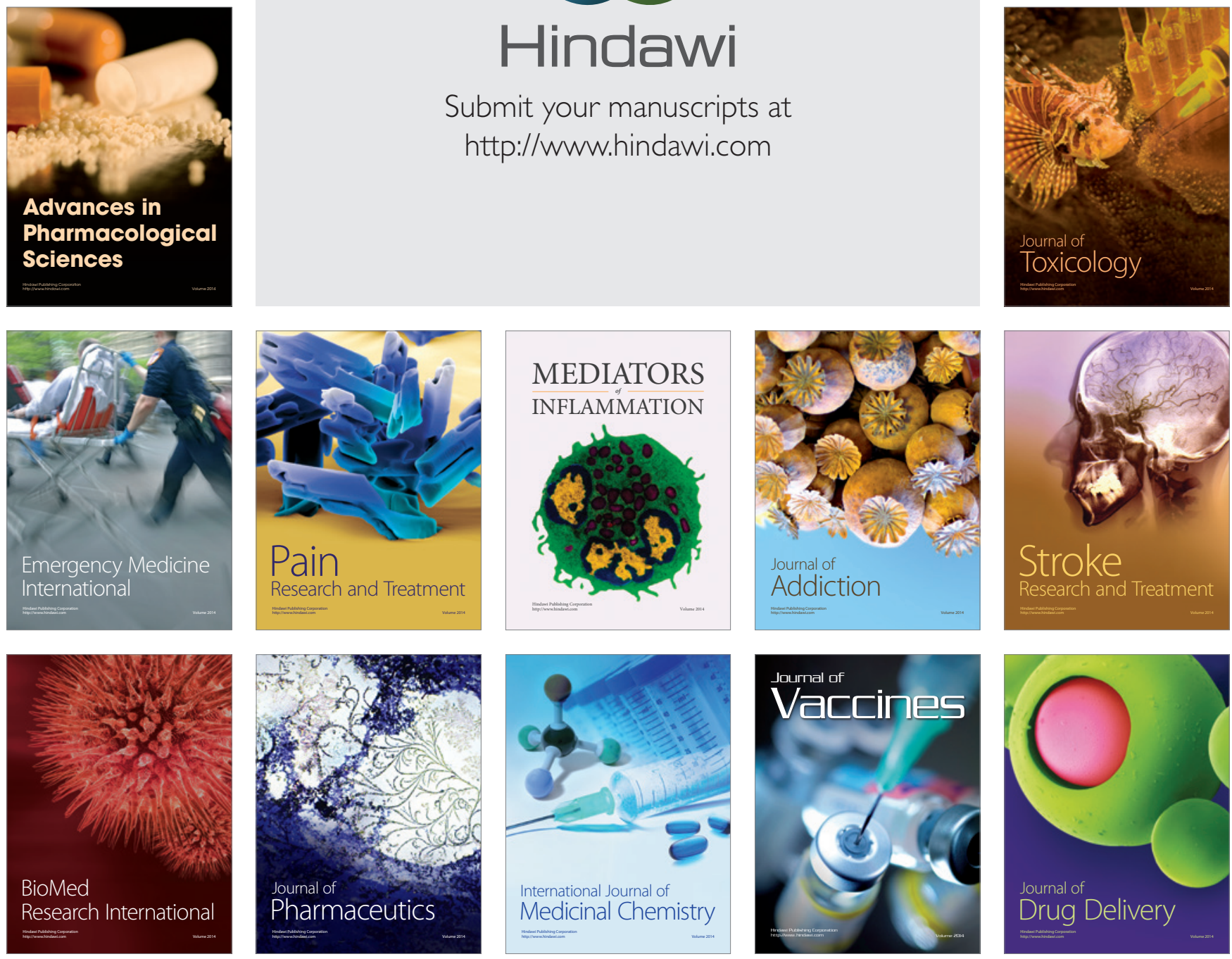